\title{
PODER Y PANOPTISMO EN EL SEGUNDO MICHEL FOUCAULT ${ }^{1}$
}

Olaya Fernández Guerrero (Universidad de La Rioja) ${ }^{2}$

olaya.fernandez@unirioja.es

Resumen: Este artículo presenta algunos aspectos de la noción de 'panoptismo', partiendo de las consideraciones y reflexiones del filósofo francés Michel Foucault, particularmente en los libros que publicó en el periodo comprendido entre 1971 y 1976, junto con las conferencias que pronunció en el Collège de France también en esos años. Asimismo, la discusión se detendrá en el momento actual, mostrando que el panoptismo ha generado prácticas de poder, reglas, normas y hábitos que forman parte de nuestra vida cotidiana y que interfieren profundamente en las maneras de actuar, relacionarse y producir subjetividades y sujeciones.

Palabras clave: disciplina, Foucault, panoptismo, poder, política.

\section{INTRODUGGIÓN: LA GENEALOGÍA DEL PODER Y LA NOCIÓN DE PANOPTISMO}

En su segunda etapa, que va desde 1971 hasta 1976, Foucault desarrolla un pensamiento filosófico orientado hacia cuestiones relacionadas con la genealogía del poder, identificando los elementos que contribuyen a crearlo, reforzarlo y reproducirlo. El análisis foucaultiano de estos años pretende identificar las prácticas divisorias y los procedimien-

\footnotetext{
${ }^{1}$ Recebido: 08-10-2017/ Aceito: 10-12-2017/ Publicado online: 08/02/2018.

${ }^{2}$ Olaya Fernández Guerrero é Professora de Filosofía da Universidad de La Rioja, Logroño, La Rioja, Espanha.
} 
tos estratégicos puesto en juego por los poderes hegemónicos, y evidencia el interés de este autor por "la descripción de las grandes estructuras de dominación y de las instituciones vinculadas a estas" (CASTELO BRANCO 2001, p. 240). En este sentido Miguel Morey ha identificado tres elementos de poder que son centrales para Foucault, y que pueden clasificarse según la siguiente tabla (MOREY 2014, p. 305):

\begin{tabular}{|l|l|l|}
\hline Dominio & Objetivo & Procedimiento \\
\hline Una óptica & Vigilancia generalizada & Panóptico \\
\hline Una mecánica & $\begin{array}{l}\text { Utilización óptica de cuerpos y } \\
\text { fuerzas }\end{array}$ & Disciplina \\
\hline Una fisiología & $\begin{array}{l}\text { Establecimiento de un cuerpo de } \\
\text { normas }\end{array}$ & Normalización \\
\hline
\end{tabular}

Desde esta perspectiva se afirma que el poder no existe únicamente en las instituciones del Estado, sino que se expande mucho más allá de los límites de lo institucional y de lo instituido. Así, para comprender bien su funcionamiento es necesario prestar atención al hecho de que el poder abarca un espacio más amplio y que se desarrolla en un terreno más ambiguo, porque "en el fondo cada uno es titular de un determinado poder y, en cierta medida, vehicula el poder" (FOUCAULT 1999, p. 321). En general, Foucault percibe que "el poder no es esencialmente represivo; que se ejerce más que se posee; y que atraviesa tanto a dominantes como a dominados" (CORTÉS 2010, p. 101-102). El poder se entiende como una multiplicidad compleja de rela- 
ciones jerárquicas en la que todas las personas están inmersas, de modo que cada cual ejerce distintos papeles de dominación y/o de sumisión dentro de ese entramado. Es por ello que el poder no ha de verse como una pertenencia que alguien en concreto posee permanentemente, sino que más bien es una fuerza, una dinámica relacional que emerge y es inherente a todo proceso de interacción intersubjetiva.

El panoptismo ocupa un lugar importante en estas reflexiones sobre el poder, ya que opera como un dispositivo de control que contribuye a reforzar, e incluso modificar, las formas en que este se ejerce en las sociedades modernas. $\mathrm{El}$ origen del panóptico se sitúa en la Inglaterra de finales del siglo XVIII, cuando Jeremy Bentham diseña un proyecto de edificio panóptico "que se convertirá en la matriz arquitectónica de las prisiones europeas" (FOUCAULT 2013, p. 66). Pero su uso no se va a circunscribir al ámbito carcelario; el panóptico, según la aproximación foucaultiana, no es simplemente un modelo de prisión, sino que "es una forma para cualquier institución; digamos, mejor, para toda una serie de instituciones" (FOUCAULT 2005, p. 84) que van a adoptar también ese tipo de estructura organizativa. El propio Bentham está de acuerdo en que, más allá de una manera de distribución espacial, el panóptico es "un mecanismo, un esquema que da fuerza a toda institución" (FOUCAULT 2005, p. 84). La mirada es el elemento central que da solidez y efectividad a este dispositivo que instaura un régimen concreto de visibilidad enfocado hacia un triple objetivo: "lograr que la fuerza del poder sea la más intensa; su distribución, la mejor, y su punto de aplicación, el más justo" (FOUCAULT 2005, P. 84). El panoptismo es una excelente manifestación del espíritu utilitarista, ya que su implantación permite una máxima eficacia con los mí- 
nimos recursos. Así, la estructura panóptica acaba por emplearse también en hospitales, escuelas o cuarteles militares; esto es, en cualquier espacio en el que se busque recluir y vigilar, con diversos fines, a las personas que están en esos edificios. El régimen panóptico, en definitiva, fortalece tanto a la institución como al individuo o individuos que, alternativamente, administran y ejercen el poder.

'Panóptico' significa dos cosas: que todo es observado todo el tiempo, y que el poder no es más que un efecto óptico. La dominación basada en este nuevo elemento ya no va a necesitar recurrir a la violencia explícita, al castigo o a la amenaza, porque el mero sometimiento a un régimen panóptico logra por sí solo que el individuo cumpla las normas y desarrolle los patrones de conducta que se esperan de él. Foucault compara este tipo de poder con el de la luz del sol, que ilumina indistintamente a todas las personas (2005, P. 88). Este dispositivo de visibilidad crea y organiza unidades espaciales que posibilitan mirar y reconocer cada detalle del campo visual. De este modo se invierte completamente la noción de calabozo, donde el prisionero permanecía a oscuras la mayor parte del tiempo. La prisión panóptica supone un cambio radical en este sentido, ya que se basa en el principio de que "la plena luz y la mirada de un vigilante captan mejor que la sombra, que en último término protegía. La visibilidad es una trampa" (FOUCAULT 1990, p. 204), puesto que nada puede escapar de la mirada que vigila y que castiga. En síntesis, en la época moderna la instauración de un régimen carcelario basado en la luz modificó sustancialmente la manera de entender las nociones de vigilancia, seguridad y control, y las huellas de esa transformación perduran hasta hoy.

En el edificio panóptico no hay ningún aspecto de las 
acciones de la persona que consiga apartarse y sustraerse al campo de lo visible. El resultado es que se teje una trama de poder que incluye "un saber permanente sobre el individuo" (FOUCAULT 2005, p. 88). Esto se debe al hecho de que la iluminación constante crea un territorio de producción de saberes sobre el individuo, en tanto que el centro de poder (la torre de vigilancia del panóptico) es también un centro de anotación y registro ininterrumpido del comportamiento individual. El panóptico es un instrumento de individuación y de conocimiento, y ambos procesos surgen simultáneamente; en el régimen panóptico se lleva a cabo un cuidadoso control y registro de todas las actividades y facetas del individuo, se produce un saber exhaustivo sobre este, y ese conocimiento omnisciente viene posibilitado precisamente por el hecho de que cada individuo está sometido a una visibilidad total y continua.

Para completar esta caracterización del panóptico, es interesante señalar otro rasgo que tiene que ver con la automatización y despersonalización. En este régimen disciplinar, cualquier persona puede ocupar el puesto de vigilante y hacer la observación (CORTÉS 2010, p. 72). Esto implica una ruptura con respecto a otras épocas anteriores, en las que la dominación era ejercida en exclusiva por un jefe claramente visible e identificable que desplegaba su liderazgo ante un conjunto de personas dominadas. El ejemplo paradigmático de este tipo de poder es la figura del emperador, que en clave visual es descrito como "el ojo universal dirigido a la sociedad en toda su extensión, un ojo que está asistido por toda una serie de miradas dispuestas de forma piramidal" (FOUCAULT 1999, p. 242). El po- 
der máximo corresponde únicamente al emperador y nadie puede suplantar su figura ni sustituirle en su ejercicio ${ }^{3}$. En la época moderna, la adopción del modelo panóptico va a trastocar completamente esta jerarquía, pues a partir de entonces la administración de la mirada no será prerrogativa de un único individuo investido de poder, sino que habrá una alternancia y una ubicuidad de los campos de visibilidad, una multiplicidad de ámbitos de vigilancia y una proliferación de individuos que pueden relevarse en la administración y regulación de esos ámbitos. El panoptismo trae consigo un poder más difuso y fluido, imposible de asociar a una sola persona que actúe como foco y origen de la dominación.

\section{VISIBILIDAD Y GONTROL}

La creciente aplicación del panoptismo a partir de finales del siglo XVIII ha conllevado un cambio radical en los modos de distribución del poder. Deleuze señala que, a partir de ese momento, el principal objetivo de quien quiera ejercer la dominación será conseguir "que la visibilidad se extienda a toda la sociedad en lugar de concentrarse en un punto. Lo que se pretende, por tanto, es una visibilidad extendida y general" (DELEUZE 2014, p. 126). Las fronteras de ese nuevo poder óptico, de ese régimen basado en la mirada, son mucho más porosas que las que habían existido en etapas anteriores, y por ello más difíciles de identificar y delimitar con exactitud.

\footnotetext{
${ }^{3}$ En la mitología griega, esa figura de máximo poder basado en la capacidad de verlo todo y de castigarlo todo está representada por Zeus, cuya dominación puede ser interpretada en términos de panoptismo. Cf. FERNÁNDEZ, O. Mirada y poder. Una interpretación post-estructuralista del mito de Perseo. Anales del Seminario de Historia de la Filosofía, v. 32, n 2, p. 543-565, 2015.
} 
Foucault define la situación del individuo aprisionado en el panóptico como una sumisión a un estado de visibilidad permanente (FOUCAULT 2005, p. 87). Además, es a través de esa visibilidad que el poder asegura su efecto (DELEUZE 2014, p. 128), en la medida en que el sujeto se sabe bajo esa mirada constante y percibe la dominación que el régimen de vigilancia le impone a todas horas. Como resultado, el poder visual así ejercido acaba por bloquear y neutralizar cualquier intento de transgredir la norma, instaura una vaga y sutil amenaza de castigo que, en la mayor parte de las ocasiones, ni siquiera necesita materializarse. En el régimen panóptico la presencia del vigilante, o incluso la simple sospecha sobre la posibilidad de su presencia, ya es suficiente para garantizar un funcionamiento exitoso del dispositivo de poder. En ese nuevo escenario ya no es preciso vigilar y castigar de facto a quien transgreda las reglas, sino que basta con aplicar una supervisión de tipo panóptico que sitúa y somete todo a una visibilidad total, hasta el punto de que se hace innecesario el castigo directo, que adquiere carácter anecdótico.

El panóptico, inicialmente basado en el modelo militar, hace surgir un tipo de vigilancia que va a aplicarse también en otros espacios de reclusión y control aparte de las prisiones para las que fue concebido en un principio. "La arquitectura de los hospitales, escuelas, cuarteles trata de ajustarse a este modelo de visibilidad (interior) total" (MOREY 2014, p. 345), utilizando así el diseño utópico de la cárcel ideal de J. Bentham para la creación de otros edificios en los que los individuos residen, trabajan y/o permanecen recluidos en distintos momentos de sus vidas cotidianas. Un rasgo común a todos esos espacios es la división en células individuales, es decir, la particularización y 
separación de los individuos: cada uno de ellos es apartado de todos los demás a través de la visibilidad que, aunque sea aplicada colectiva y homogéneamente a todos ellos, los aísla y los convierte en sujetos - sujetos a la imposición de la mirada vigilante. En la escuela, en el hospital o en el cuartel hay una supervisión permanente de cada persona concreta, un seguimiento detallado de los procesos de aprendizaje, de las conductas sociales, de los estados de salud, del cumplimiento de las normas... y todo ello es posible precisamente por el recurso a esa visibilidad poderosa y omnipresente que el régimen panóptico pone en juego.

En la sociedad panóptica el control no adopta la forma de una comprobación ni de un interrogatorio, más bien se parece a un examen sin fin, a un cuestionamiento previo a la comisión de cualquier delito, y donde toda persona es a priori sospechosa. La idea de culpabilidad, basada en la constatación de la realización de ciertas acciones por parte de un determinado sujeto, es sustituida por la noción de 'peligrosidad', mucho más capciosa y resbaladiza, ya que implica "que el individuo debe ser considerado por la sociedad en razón de sus virtualidades y no tanto en razón de sus actos" (FOUCAULT 1999, p. 226). En ese nuevo contexto se aplica una "prueba ininterrumpida, gradual, acumulativa, que permite un control y una presión sobre todos los instantes, de seguir al individuo en cada uno de sus procesos, de ver si es regular o irregular, ordenado o disipado, normal o anormal" (FOUCAULT 2013, p. 201). Bajo este régimen visual todo acto de la persona es observado $\mathrm{y}$ apuntado, de manera que la visibilidad, instituida como elemento de control, influye decisivamente en la producción de subjetividades. El sujeto se constituye y articula precisamente en el medio de esa interacción de fuerzas, mi- 
radas y dispositivos de poder que definen y dan lugar a la sociedad disciplinar, de la que se hablará con más detalle posteriormente.

El individuo que está sometido a un campo de visibilidad panóptica acaba por aceptar e interiorizar esa situación con tal intensidad que, en último término, "reproduce por su cuenta las coacciones del poder; las hace jugar espontáneamente sobre sí mismo; inscribe en sí mismo la relación de poder en la cual juega simultáneamente los dos papeles; se convierte en el principio de su propio sometimiento" (FOUCAULT 1990, p. 206), y aquí reside para nuestro autor el mayor éxito, la máxima efectividad de este dispositivo de poder que acaba entrando en la psique del individuo, provoca que el sujeto crea ciegamente en la vigilancia constante que pesa sobre él, y hace que cada cual se convierta en su propio carcelero.

\section{VISIBILIDAD Y PRODUGGIÓN DISGURSIVA}

Foucault reitera que "el poder nos somete a la producción de la verdad y sólo podemos ejercer el poder por la producción de la verdad" (2003, p. 30). A partir de ahí se deduce que el funcionamiento de cualquier dispositivo de poder siempre lleva implícita la puesta en marcha de distintos procesos de producción discursiva, y el panoptismo no es ajeno a esta circunstancia. Nuestro autor aborda el estudio de la verdad como el conjunto "de técnicas conducentes a afianzar la subjetividad como construcción y como experiencia" (FORTANET 2012, p. 102), y en ese escenario se gesta una conexión íntima entre la mirada y la palabra, entre lo visible y las descripciones y elaboraciones discursivas que dan cuenta de lo visto. La visibilidad guarda una estre- 
cha relación con el lenguaje, en la medida en que "el saber es un 'dispositivo' de enunciados y visibilidades" (DELEUZE 2004, p. 58) que produce la verdad a través del lenguaje y que, mediante ese mecanismo, crea 'etiquetas' y fija las categorías a partir de las que se percibe y conceptualiza la realidad. En la construcción de los saberes, el lenguaje opera como una retícula en la que se van insertando diferentes elementos, ajustándose a cada una de las celdas de esa matriz discursiva. "El saber entrelaza luz y lenguaje" (DELEUZE 2014, p. 47), y la mirada y la palabra están interconectadas hasta el punto de que los regímenes de visibilidad y los regímenes de discursividad confluyen en muchas de las prácticas de poder que realizamos y/o padecemos cotidianamente, según se refleja en el análisis foucaultiano. El ojo y la palabra son los dos elementos que interactúan y cooperan entre sí para dar lugar a la 'verdad', es decir, para producir los discursos que enuncian lo visible y le confieren inteligibilidad.

Partiendo de estas tesis se entiende que uno de los rasgos más importantes de la sociedad basada en el panoptismo, a la que Foucault denomina 'sociedad disciplinar', sea el establecimiento de una vigilancia general que da lugar al despliegue de un saber lingüístico y discursivo sobre los individuos, que son "sometidos a un constante examen" (FOUCAULT 2013, p. 200). La aplicación del principio panóptico "efectúa una individualización centralizada cuyo soporte e instrumento es la escritura" (FOUCAULT 2005, p. 63). En toda institución basada en este fundamento - escuela, hospital, prisión, cuartel militar - la elaboración de informes, historiales, fichas, expedientes, etcétera, forma parte del quehacer cotidiano, y quienes ejercen la vigilancia y administran disciplina en cualquiera de esos es- 
pacios cuentan entre sus tareas y obligaciones con las de llevar un registro exhaustivo y por escrito de todas las actividades, conductas, incidentes, que atañen a los individuos ligados a esas instituciones. En el régimen panóptico, los sujetos están vinculados simultáneamente a la visibilidad y a la discursividad, puesto que, mientras son observados, se registran minuciosamente por escrito los resultados de esa observación.

La mirada y la palabra homogeneizan y conectan entre sí a todos los individuos que "están insertos en un lugar fijo, en el que los menores movimientos se hallan controlados, en el que todos los acontecimientos están registrados, en el que un trabajo ininterrumpido de escritura une el centro y la periferia" (FOUCAULT 1990, p. 201). El campo visual es también un campo de producción discursiva que articula y construye conocimientos sobre los individuos sometidos a un régimen de visibilidad. Además, dado que se despliega una mirada panóptica, nada queda fuera de la vigilancia ni consigue esconderse del órgano (ocular) de poder que analiza y registra cada variación, cada mínimo cambio: "El centro del poder es al mismo tiempo un centro de anotación ininterrumpida, de anotación del comportamiento individual" (FOUCAULT 2005, p. 88).

Así las cosas, ya no hay absolutamente ningún aspecto que permanezca oculto, y esto acaba provocando que la distinción entre centro y periferia pierda sentido; en el régimen panóptico el único centro que existe es el de la mirada-poder, el ojo escrutador que controla y supervisa lo que está a su alrededor y que deja constancia escrita de todo lo que acontece en cada uno de los puntos del espacio, en cada hilo y en cada extremo de esa tupida red que conforma el ámbito de lo visible. 
En ese contexto, y como resultado de todo lo expuesto, surge un nuevo tipo de saber basado en la vigilancia y el examen y enfocado fundamentalmente hacia el control de los individuos. Foucault considera que esta práctica abre el camino para la creación y consolidación de las denominadas 'ciencias humanas', la psiquiatría, psicología o sociología (1999, p. 229), ya que todas ellas arrancan de la observación y el registro escrito de las conductas individuales o colectivas, para posteriormente elaborar saberes, conocimientos y pautas de intervención que buscan interferir de diferentes maneras en algunas de esas conductas y que tratan de modificarlas en caso de que estas sean consideradas 'patológicas' o 'anormales', es decir, desviadas de la norma.

La producción discursiva que se despliega en el régimen panóptico da lugar también a una nueva modalidad de poder que se perfila como un 'poder epistemológico', "capaz de extraer de los individuos un saber, un saber que además se refiere a estos propios individuos sometidos a la mirada" (FOUCAULT 1999, p. 253). La efectividad y exhaustividad de ese poder omnipresente y omnipotente que es inherente al panoptismo solo se entiende bien si se tienen en cuenta ambas dimensiones, la mirada y la palabra, y si se explica el poder como resultado de la conjugación y cooperación de estos dos elementos. Los individuos son al mismo tiempo "aquello a partir de lo que se va a extraer el saber que ellos mismos formaron [...], o bien objetos de un saber que permitirá también, igualmente, nuevas formas de control" (FOUCAULT 1999, p. 254). La generación de saberes sobre los individuos da lugar a normas, pautas y reglas que se van a aplicar directamente sobre esos mismos individuos, reforzando y consolidando de este modo su su- 
jeción al sistema de vigilancia panóptica. "Ese poder inmaterial perpetuamente ejercido a través de la iluminación está ligado a una extracción permanente de saber" (FOUCAULT 2005, p. 88), el individuo en todas sus facetas y rasgos se convierte en objeto principal de conocimiento; cada sujeto concreto, paradójicamente, acaba por constituirse en contenido de un saber que está primordialmente enfocado a optimizar los mecanismos que garantizan su sometimiento y dominación.

De hecho, y llevando esta argumentación hasta sus últimas consecuencias, Foucault considera que no puede concebirse al individuo al margen de las relaciones de poder en que está inmerso, ni prescindiendo de los saberes y conocimientos que se producen sobre él en el seno de esas relaciones. La subjetividad y la identidad surgen como resultado de la incardinación en ese entramado, puesto que "el individuo se constituyó en la medida en que la vigilancia ininterrumpida, la escritura continua y el castigo virtual dieron marco a ese cuerpo así sojuzgado y le extrajeron una psique" (FOUCAULT 2005, p. 67). La visibilidad y la enunciabilidad son, en último término, los campos que establecen la inteligibilidad de los sujetos, y no se puede comprender la emergencia de lo psíquico sin tomar en consideración esas determinaciones. De esta manera surge la paradoja, señalada por Judith Butler, de que "el poder es a la vez externo al sujeto y el propio lugar del sujeto" (BUTLER 1997, p. 15), sin que sea fácil deslindar dónde empieza y dónde acaba la interioridad y la exterioridad con respecto a ese poder.

En el régimen panóptico hay una plena conexión entre los mecanismos de producción de subjetividades y la creación de saberes con respecto a esas subjetividades. El tér- 
mino 'sujeto' designa al individuo tomado como objeto dominado por las prácticas de poder, de tal manera que "las verdades reconocidas en el sujeto por conocer son tributarias de una serie de prácticas de poder que posibilitan ciertos tipos de conocimiento y de verdad" (SOUZA 2010, p. 12); los mecanismos y resortes del poder generan subjetividades a la vez que hacen posible el acceso epistemológico a esas subjetividades. De hecho, Foucault describe el panóptico como "un aparato de individualización y conocimiento a la vez; un aparato de saber y poder a la vez, que individualiza por un lado $\mathrm{y}$, al individualizar, conoce" (FOUCAULT 2005, p. 88), consolidando así el vínculo entre mirada, discurso y poder. El campo de lo visible es descrito con palabras y entonces deviene campo de enunciación, se articula como ámbito de lo expresable; ese poder visual y lingüístico se inscribe sobre los cuerpos de los individuos y acaba penetrando también hasta lo más profundo de su subjetividad.

\section{LA SOCIEDAD DISGIPLINAR}

Partiendo del análisis genealógico del poder planteado por Foucault, el período contemporáneo puede calificarse de 'sociedad disciplinar'. Su rasgo más destacado es el surgimiento de una nueva modalidad de poder que consigue finalmente entrar en la interioridad de los cuerpos y de las psiques, abarcando cada uno de los intersticios de la vida material e inmaterial de los individuos, e influyendo incluso en las conductas, pensamientos, palabras y sentimientos. Emerge entonces una forma de poder muy específica basada en un "contacto sináptico cuerpo-poder" (FOUCAULT 2005 , p. 51) que va a caracterizar, de manera general, to- 
das las actividades y dispositivos de control de la vida que se incluyen en el 'biopoder', a cuyo análisis Foucault se dedica de lleno en sus últimas obras.

La sociedad disciplinar, surgida a partir del siglo XVIII, despliega una dominación ética y política enfocada hacia el objetivo de que "el cuerpo, el tiempo, la vida, los hombres se integren, bajo la forma del trabajo, en el juego de las fuerzas productivas" (FOUCAULT 2013, p. 201). No es casual, para este filósofo, que ese modelo de sociedad surja al mismo tiempo que el capitalismo. De hecho, Foucault considera que todos los dispositivos y mecanismos específicos del poder disciplinar contribuyen a reforzar y promover la adaptación y sujeción de los individuos al sistema productivo capitalista. En ese contexto, el poder se apropia de las fuerzas de los individuos y entra en sus vidas para hacer que esas fuerzas no se pierdan y desperdicien en actividades improductivas. Su lógica es que todos los ámbitos de la subjetividad, sin excepción, han de ser controlados y enfocados hacia la utilidad y la productividad, calculadas, eso sí, en términos exclusivamente económicos.

La disciplina persigue el objetivo de dominar a la multiplicidad de los sujetos, para ello ejerce "una coerción ininterrumpida" (MOREY 2014, p. 342) que se aplica a todos los procesos corporales y psíquicos, en un intento de controlar exhaustivamente cada uno de estos. Para alcanzar esta meta, la sociedad disciplinar necesita en primer lugar establecer variables discretas, "debe resolverse en cuerpos individuales que hay que vigilar, adiestrar, utilizar y, eventualmente, castigar" (FOUCAULT 2003, p. 208). El panoptismo sirve de manera óptima a este objetivo, en tanto que su uso garantiza una vigilancia eficaz y efectiva de los cuerpos sometidos a la dominación. El propio Foucault 
admite que la invención de Bentham "presenta con toda exactitud la fórmula política y técnica más general del poder disciplinario" (FOUCAULT 2005, p. 52), ya que permite instaurar un régimen basado en la visibilidad y orientado hacia la disposición de los cuerpos en el espacio de tal manera que se facilita al máximo la regulación y administración de esos cuerpos en todas sus dimensiones y facetas.

Uno de los efectos más importantes de la disciplina es la 'normalización', que alude a la aceptación e interiorización de las normas por parte de los individuos. El poder sustentado en el tándem disciplina-normalización no es represivo, no necesita ejercer una violencia y coacción constantes sobre el sujeto para conseguir que este respete las reglas, sino que su funcionamiento es de carácter productivo. La eficacia de ese poder va a depender mayoritariamente de los "mecanismos que fabrican, mecanismos que crean, mecanismos que producen" (FOUCAULT 2001, p. 56). Dentro del amplio abanico de dispositivos de producción y creación material y discursiva de las subjetividades, la visibilidad panóptica tiene un papel protagonista, como ya se ha expuesto anteriormente. En ese contexto, la díada 'vigilarcastigar' se erige en "relación de poder indispensable para la fijación de los individuos al aparato de producción, a la constitución de las fuerzas productivas" (FOUCAULT 2013, p. 201). En definitiva, la supervisión panóptica y la amenaza difusa de castigo en el caso de incumplir las reglas son el elemento de poder más característico de la sociedad disciplinar.

Según este planteamiento, el sujeto surge como resultado y producto de la sociedad disciplinar. La identidad de cada persona es un efecto del poder que solamente aparece 
al final de un 'procedimiento de individualización' proporcionado y administrado por el propio poder. " $\mathrm{El}$ individuo, el grupo, la colectividad, la institución, aparecen contra el fondo de esa red de poder, y funcionan en sus diferencias de potencial y sus desvíos" (FOUCAULT 2005, p. 28). Partiendo de esta premisa, el estudio de las instituciones y la comprensión de su funcionamiento deben ir precedidos de un análisis de las relaciones de poder, de las distintas fuerzas e intensidades que envuelven a los individuos y marcan el posicionamiento de estos en cada una de esas instituciones. Las disciplinas crean diferentes estereotipos de 'persona normal', que es producida a partir de y como resultado de los variados regímenes de control y vigilancia desplegados por las distintas instituciones por las que el individuo va pasando a lo largo de su existencia (MOREY 2014, p. 361). Por ejemplo, la escuela, el hospital o el cuartel militar son, respectivamente, los espacios que definen al estudiante, al enfermo, al soldado, de modo que únicamente en el contexto de esas instituciones adquieren su significado esos términos lingüísticos empleados para definir las identidades individuales.

Los procesos de normalización de la conducta inherentes a la sociedad disciplinar consisten en "una transformación técnica de los individuos para adecuarlos a una norma: fabrica hombres normales" (MOREY 2014, p. 348), pero ese mecanismo también tiene sus puntos débiles, ya que no todos los sujetos consiguen adaptarse completamente a todas las reglas que la sociedad establece a priori. Por este motivo existen subjetividades hegemónicas, más próximas a lo que las normas definen, y subjetividades marginales, que no terminan de encajar en los cánones predeterminados. Así, acontece que "en el sistema disciplinario 
el principio de distribución y clasificación de todos los elementos implica necesariamente un residuo; siempre hay, entonces, algo 'inclasificable"” (FOUCAULT 2005, p. 64), algún aspecto de la conducta individual que permanece al margen de la norma y que, por eso mismo, supone una amenaza y un cuestionamiento de esa norma, de modo que esa transgresión será vista con recelo por parte del poder. Asimismo, en la sociedad disciplinar el recurso a la vigilancia panóptica hace posible señalar exactamente cuáles son las identidades que se apartan de la norma, e incluso definir el grado de desviación que cada individuo tiene con respecto a cada pauta concreta de entre todas las que regulan y fijan los códigos de la conducta social, sexual, profesional, religiosa, etcétera, considerados aceptables en esa sociedad.

\section{AlgunAS GONGLUSIONES: EL PANOPTISMO EN NUESTRO TIEMPO}

Después de todo lo expuesto, es evidente que muchos de los rasgos con los que Foucault describe el panoptismo tienen presencia en nuestras sociedades del siglo XXI. El Panóptico, máquina política ideal, nació con una voluntad expansiva: "el esquema panóptico, sin anularse ni perder ninguna de sus propiedades, está destinado a difundirse en el cuerpo social; su vocación es volverse en él una función generalizada" (FOUCAULT 1990, p. 211). El panoptismo es la manera específica en la que la época actual ejerce y desenvuelve su dominación sobre la fuerza productiva de los seres humanos; aparece también como el modo más eficaz de control de la producción de saberes sobre esos seres humanos en tanto que individuos. "Vivimos en el panoptismo generalizado por el hecho mismo de vivir dentro de un sis- 
tema disciplinario" (FOUCAULT 2005, p. 90). En la época actual, la disciplina es panóptica, en tanto que todos los individuos sin excepción son disciplinados y producidos en los diversos campos de visibilidad más o menos sutil que forman parte de la vida cotidiana. Cada actuación y cada movimiento en la escuela, en el lugar de trabajo, en las calles, en los aeropuertos, en la cuenta bancaria, en el teléfono móvil o en el muro de Facebook están sujetos a una supervisión constante, a una vigilancia total que se aproxima peligrosamente a la utopía panóptica. El propio Foucault corrobora esta tesis cuando afirma que "el panóptico es la utopía de una sociedad y de un tipo de poder que, en el fondo, es la sociedad que conocemos actualmente" (1999, p. 228). El reciente avance de las tecnologías de control, el régimen de vigilancia global que se ha incrementado en este siglo, e incluso la aceptación acrítica del panoptismo por parte de todos los individuos que comparten hasta el más mínimo detalle de sus vidas personales en sus cuentas de Instagram o de Twitter, vienen a confirmar este diagnóstico que Foucault realizó hace casi cuarenta años.

El panoptismo está hoy muy presente en las instituciones que gobiernan las vidas y los cuerpos de los individuos, tejiendo el telón de fondo del escenario en el que transcurre nuestra existencia. La visibilidad radical, en conexión con el poder, entra en las vidas concretas, influyendo en ellas de un modo tan efectivo y exitoso que consigue irrumpir en todas las acciones, ideas, movimientos y sentimientos del sujeto, y resulta difícil evadirse de ese control y plantear actitudes y propuestas de resistencia frente a él.

En las sociedades actuales el poder solamente es comprensible como la combinación y el resultado de la alternancia "entre un derecho público de la soberanía y una 
mecánica polimorfa de la disciplina" (FOUCAULT 2003, p. 41), los dos grandes ámbitos de poder que se refuerzan y retroalimentan. Más allá de las leyes recogidas y objetivadas en los textos jurídicos, y que son fácilmente identificables, los cuerpos y las fuerzas están también sometidos a múltiples dispositivos de 'encarcelamiento', control y sujeción; nuestras vidas están envueltas en un amplio abanico de regímenes disciplinares, mayoritariamente visuales, que administran y crean burocracias que afectan sin excepción a todas y cada una de las actividades de los individuos. El resultado es una "humanidad central y centralizada, efecto e instrumento de relaciones de poder complejas" (FOUCAULT 1990, p. 314) que, para ser bien comprendidas, han de ser estudiadas genealógicamente.

Todas las instituciones sociales - fábrica, escuela, hospital psiquiátrico, prisión - son espacios de administración disciplinar, de gestión y producción de subjetividades, y su finalidad no es excluir sino, al contrario, "fijar a los individuos" (FOUCAULT 1999, p. 248), afianzando la sujeción de estos a las reglas, normas y códigos de conducta considerados 'normales' en cada sociedad concreta. El uso y aplicación del modelo panóptico en todo el cuerpo social ha contribuido a cambiar las formas en que se establecen y hacen cumplir las disciplinas, hasta el punto de que el panóptico produce "la normalización del estado de sitio" (MOREY 2014, p. 356). En el siglo XXI es considerado 'normal' que exista un control y seguimiento exhaustivo de los movimientos de la población, y en muchas ocasiones, bajo el pretexto de garantizar la seguridad, son vigiladas las llamadas telefónicas, las gestiones bancarias, los viajes que cada persona realiza, e incluso los libros que toma prestados de la biblioteca pública. En la sociedad actual parece 
que cualquier persona puede súbitamente ser sospechosa de algún delito, y que todo su entorno actúa como guardián y tiene la potestad de señalar a esa persona en cualquier instante y denunciar su conducta, en caso de que esta no se ajuste a las reglas previamente establecidas por esa sociedad. Esta situación, vivida cotidianamente, evoca la descripción del panoptismo planteada por Foucault.

Así, las reflexiones de este filósofo francés resultan de utilidad para entender mejor algunos de los procesos y rasgos más característicos de nuestro tiempo, particularmente en lo que tiene que ver con los nuevos tipos de dominación $\mathrm{y}$ con las relaciones y posiciones de poder que condicionan los procesos de articulación y exégesis de las identidades de los sujetos contemporáneos. El panoptismo es uno de los elementos más representativos de la sociedad actual, y Foucault ofrece elementos valiosos para comprender en profundidad tanto sus mecanismos como sus efectos.

\begin{abstract}
This article presents some aspects of the notion of 'panoptism', starting from the considerations and reflections of the French philosopher Michel Foucault, particularly in the books he published in the period between 1971 and 1976, together with the lectures given at the Collège de France also in those years. Moreover, the discussion will stop at the present moment, showing that panopticism has generated practices of power, rules, uses and habits that are part of our daily life and that deeply interfere in the ways of acting, relating and producing subjectivities and subjections.
\end{abstract}

Keywords: discipline, Foucault, panopticism, power, politics.

\title{
REFERENCIAS
}

BUTLER, J. The Psychic Life of Power. Theories in Subjection. California: Stanford University Press, 1997.

CASTELO BRANCO, G. As resistências ao poder em 
Michel Foucault. Trans/form/ação, n. 24, p. 237-248, 2001.

Cortés Rodríguez, M. Á. Poder y resistencia en la filosofia de Michel Foucault. Madrid: Biblioteca Nueva, 2010.

DELEUZE, G. Michel Foucault y el poder. Madrid: Errata Naturae, 2014.

Deleuze, G. Foucault. Paris : Éditions de Minuit, 2004.

FORTANET, J. Experiencia, ética y poder en la obra de Michel Foucault. Oxímora. Revista internacional de ética y política, n 1, p. 96-114, otoño 2012.

Foucault, M. La societé punitive. Cours au Collège de France (1972-1973). Paris: Seuil-Gallimard, 2013.

FOUCAULT, M. El poder psiquiátrico. Curso del Collège de France (1973-1974). Madrid: Akal, 2005.

Foucault, M. Los anormales. Curso del Collège de France (1974-1975). Madrid: Akal, 2001.

Foucault, M. Hay que defender la sociedad. Curso del Collège de France (1975-1976). Madrid: Akal, 2003.

FOUCAULT, M. Vigilar y castigar. Nacimiento de la prisión. Madrid: Siglo XXI, 1990.

FOUCAULT, M. Estrategias de poder. Barcelona: Paidós, 1999.

MOREY, M. Lectura de Foucault. Madrid: Sexto Piso, 2014.

SOUZA, C. de. Vontade e verdade em Foucault. Philóso- 
phos, v. 15, n. 2, p. 11-28, jul./dez. 2010.

N. B. Este artigo faz parte do projeto de pesquisa "Gobierno de sí y políticas de la subjetividad en el contexto de la crisis de la racionalidad neoliberal" (FFI2016-76856- R), financiado pelo Governo da Espanha.

https://filosofia.unizar.es/gobierno-de-si-y-politicas-de-lasubjetividad 